\title{
Lumen
}

Selected Proceedings from the Canadian Society for Eighteenth-Century Studies

\section{Morgan, le « négrillon » de Chateaubriand}

\section{Michèle Bocquillon}

Volume 38, 2019

URI : https://id.erudit.org/iderudit/1059270ar

DOI : https://doi.org/10.7202/1059270ar

Aller au sommaire du numéro

Éditeur(s)

Canadian Society for Eighteenth-Century Studies / Société canadienne d'étude du dix-huitième siècle

ISSN

1209-3696 (imprimé)

1927-8284 (numérique)

Découvrir la revue

Citer cet article

Bocquillon, M. (2019). Morgan, le « négrillon » de Chateaubriand. Lumen, 38,

15-30. https://doi.org/10.7202/1059270ar d'utilisation que vous pouvez consulter en ligne.

https://apropos.erudit.org/fr/usagers/politique-dutilisation/ 


\title{
Morgan, le «négrillon» de Chateaubriand
}

\author{
MichèLE BoCQuillon \\ City University of New York
}

En 1823 paraît Ourika, un roman de Mme de Duras dont on a beaucoup reparlé ces dernières décennies ${ }^{1}$. Je ne vais pas en proposer une nouvelle lecture, mais rappeler certains éléments de sa composante historique, car l'héroïne du roman, Ourika, a bel et bien existé. Elle fut rapportée d'Afrique à l'âge de 2 ou 3 ans par le chevalier de Boufflers, gouverneur du Sénégal de 1785 à 1787 , qui l'a offerte à son oncle le maréchal de Beauvau. La famille de Beauvau n'est pas la seule à avoir profité de la prodigalité du chevalier de Boufflers. Il a offert d'autres «négrillons²» à ses proches, à la reine et à la famille d'Orléans. Il était de bon ton au XVIII siècle dans les milieux aristocratiques de s'entourer de petits «négrillons» qui, le temps de leur enfance, distrayaient le beau monde. Mme de Genlis, «gouverneur» des enfants du duc d'Orléans, a bien rendu compte de la condition de ces enfants noirs vivant au Palais Royal dans ses écrits ${ }^{3}$. Ils sont choyés comme le sont les animaux de compagnie et «règnent» dans le salon, aussi longtemps qu'ils demeurent petits et amusants; ils sont ensuite « relégués

1. Voir Mme de Duras, Ourika, éd. Roger Little, Exeter, University of Exeter Press, 1998.

2. "NÉGRILLON, s. m. (Commerce d'Afrique.) on nomme negrillons dans le commerce des esclaves, les petits negres de l'un ou de l'autre sexe qui n'ont pas encore passé dix ans: trois enfans de dix ans sont deux pieces d'Inde, \& l'on compte deux enfans de cinq ans pour une piece» (Encyclopédie ou dictionnaire raisonné des sciences, des arts et des métiers, Neufchastel, Chez Samuel Faulche et Compagnie, 1765 , t. XI, p. 85).

3. Voir Michèle Bocquillon, «La domesticité négrillonne à Paris au siècle des Lumières », Archives et Bibliothèques de Belgique, 86, printemps 2009, p. 99-123. 
dans l'antichambre ${ }^{4}$ » pour être aussi vite remplacés par un autre petit négrillon. Ce ne fut pas le cas d'Ourika, qui fut tendrement aimée par la famille de Beauvau, si l'on en croit les souvenirs de la maréchale 5 Elle mourut malheureusement à la fleur de l'âge en 1799.

Une vingtaine d'années plus tard, Mme de Duras se met à écrire un roman mettant en scène Ourika. La conception de ce roman commence dans son salon: «En 1820 seulement, ayant un soir raconté avec détail l'anecdote réelle d'une jeune négresse élevée chez la maréchale de Beauvau, ses amis, charmés de ce récit (car elle excellait à raconter), lui dirent: "Mais pourquoi n'écririez-vous pas cette histoire?" ». L'impulsion est donnée. Selon Pailhès, "[e]lle avait dû composer Ourika, du moins en partie, [...] de janvier à avril $1821^{7}$ » et Chateaubriand en avait suivi de près la composition; il en avait lu les épreuves. Dans son Journal des années 1821-1827, Mme de Duras annonce le 8 janvier 1822 : «J'ai fait Ourika je ne sais si j’ai réussi. Cette occupation a interrompu le journal, je vais le reprendre ${ }^{8} »$. Or, à l'entrée du 20 novembre 1821 , elle avait écrit:

«[...] C’est un évén[ement] qui s'est passé de nos jour[s et dont] j'ai été témoin. Le chevalier de BouffLers avoit rapporté du Sénégal à Made la maréchal de BEAUvaU une petite négresse »... Et Mme de Duras raconte l'histoire d'Ourika, élevée au milieu de «la société la plus spirituelle de Paris », mais progressivement isolée par la couleur de sa peau: «elle en étoit venue à ne pouvoir se regarder dans un miroir. Sa figure lui paroissoit celle d'un monstre, elle devint triste, sauvage, chaque année ajoutoit à sa mélancolie. Elle mourut de chagrin avant 20 ans, et se trouva heureuse de mourir »?

\footnotetext{
5. Souvenirs de la maréchale princesse de Beauvau, Paris, Techener, 1872, p. 149150 et 162 . Voir Mme de Duras, Ourika, op. cit., p. 56.

6. Charles-Augustin Sainte-Beuve, Portraits de femmes, Paris, Garnier, 1886, p. 69, je souligne.

7. Gabriel Pailhès, La duchesse de Duras et Chateaubriand, Paris, Perrin, 1910, p. 292.

8. Mme de Duras, "Carnet autographe, 1821-1827", cité dans le catalogue de ventes aux enchères publiques de Pierre Bergé et Associés, La duchesse de Duras et ses amis, Chateaubriand. Autographes et manuscrits, Paris/Bruxelles, Fondation Pierre Bergé/Yves Saint-Laurent, 24 octobre 2013, n¹85, p. 140.

9. Id., je souligne.
}

4. Mme de Genlis, Souvenirs de Félicie, Paris, Librairie Firmin Didot et Cie, 1879, p. 180. 
On s'était jusqu'ici posé des questions sur la mort, non de l'Ourika de papier, mais de la petite Ourika arrivée à Paris en 1786. Mme de Beauvau n'en mentionne pas la cause dans ses Souvenirs. Dans l'entrée de son journal, Mme de Duras, qui se pose en témoin, donne ici clairement les raisons de la mort d'Ourika. À propos du roman, Chateaubriand écrit mi-décembre à Mme de Duras: «[...] en lisant les premières pages, j’ai pleuré » et «Je suis tout ému d'Ourika ${ }^{10}$ », fin décembre. Goethe, lui-même, reconnaît après avoir lu Ourika: «À mon âge, il ne faut pas se laisser émouvoir à ce point ${ }^{11}{ }^{\prime}$. Les lecteurs d'Ourika ont donc été attendris par la destinée tragique de cette enfant noire déracinée qui n’a pu, en fin de compte, trouver place dans la société française.

On pourrait dès lors penser que cette apparente sollicitude, jointe à l'abolition de la traite des Noirs ${ }^{12}$, ferait passer la mode du négrillon si répandue sous l'Ancien Régime. Or Chateaubriand a lui-même reçu un petit négrillon, nommé Morgan, de son ami Drovetti, consul général de France à Alexandrie ${ }^{13}$. Il nous le confie dans sa relation du Congrès de Vérone:

Pour nous délivrer de ce rendez-vous de mouches qui bourdonnent partout où s'épand quelques gouttes d'or, nous n'avions pas, comme l'amiral turc de M. Choiseul-Gouffier, un lion familier venant sentir aux mains de nos visiteurs $^{14}$; mais nous avions un négrillon qui leur passait entre les jambes, les tiraillait et les interrompait dans leurs discours. Il nous avait été envoyé d'Égypte par notre hôte et ami, Mr. Drovetti. Il était fils de prince; il s'appelait Morgan (la perle), nom de tendresse que lui avait donné sa mère ${ }^{15}$,

10. François-René de Chateaubriand, Correspondance générale, éd. Pierre Riberette, Paris, Gallimard, 1983, t. IV, p. 226 et 233.

11. "Lettre de Humboldt à madame de Duras, le 13 décembre 1826 ", cité par Pailhès, La duchesse de Duras, op. cit., p. 313.

12. Loi du 15 avril 1818. Cet article n'est pas le lieu d'une réflexion générale sur l'esclavage de l'époque, mais celui d'une mise en lumière d'une pratique de l'esclavage souvent passée sous silence.

13. Chateaubriand avait rencontré Drovetti en 1806 lors du voyage qu'il retrace dans Itinéraire de Paris à Jérusalem (1811).

14. «[...] de Choiseul rapporte que Hasan-Pacha, grand-amiral d'un des derniers sultans, affectionnait un Lion domestique qui le suivait partout et reposait près de son sopha» (Félix Archimède Pouchet, Zoologie classique ou histoire naturelle du règne animal, Paris, Librairie encyclopédique de Roret, 1841, p. 86).

15. Morgan est un prénom d'origine bretonne; on peut penser qu'il a été donné à l'enfant par Chateaubriand. L'attribution du prénom telle que présentée nous semble fantaisiste. 
égorgée par les soldats du pacha ${ }^{16}$. Cet enfant était à peu près de l'âge de M. le duc de Bordeaux; celui-ci admettait à ses jeux l'orphelin esclave ${ }^{17}$ privé de son trône d'ébène. Morgan n’a pas vécu; il est mort à Rome, où nous l'avions mis à la propagande ${ }^{18}$, dans l'espoir d'en faire un archevêque d'Éthiopie: il a rendu son dernier soupir à la primeur du jour, à une heure matinale comme sa vie. Morgan, la perle de sa mère, est allé parer dans le ciel cette pauvre mère. Ce petit roi noir, à l'instar du petit roi blanc, son camarade, avait été jeté par la dérision du sort à la garde de notre faiblesse. Nous aurions mieux été assis avec lui sous un palmier, aux sources du Nil, que lui, courant auprès de nous sous les fauteuils de sa Majesté très-chrétienne, à l'hôtel des affaires étrangères ${ }^{19}$.

Nous sommes en 1823, au moment où Morgan s'amuse à l'hôtel des Affaires étrangères, l'année même où fut publié Ourika ${ }^{20}$ ! Et il doit être bien jeune puisque le duc de Bordeaux est né en 1820. Quant à Chateaubriand, il doit alors être encore imprégné et «ému» de l'histoire d'Ourika dans laquelle il a été plongé durant sa rédaction. Morgan est sans doute un cadeau du pacha Méhémet-Ali à Chateaubriand, alors ministre des Affaires étrangères ${ }^{21}$. Dans une lettre du 5 février 1824 à l'amiral, comte d'Augier, Drovetti dénombre les cadeaux envoyés à cette date de la part du vice-roi (le pacha):

M. le Commandant de la corvette m'a fourni encore l'occasion favorable de faire l'envoi de huit chevaux des plus belles races d'Égypte et d'un jeune éléphant offert à S.M. et à S.A.R.M.r le Dauphin, par son Altesse le Vice-Roi. [...] M $\mathrm{M}^{\mathrm{r}}$ Perrey, qui a été présenté plusieurs fois à S.A. le

16. L’enfant a probablement été capturé durant la campagne militaire que l'Égypte mène au Soudan (1820-1822).

17. Le statut de Morgan en Égypte.

18. «La Sacrée Congrégation pour la Propagation de la Foi, fondée en 1622 par Grégoire XV» (François-René de Chateaubriand, Le congrès de Vérone, éd. JacquesAlain Sedouy, dans Euvres complètes, sous la direction de Béatrice Didier, Paris, Champion, 2014, p. 381 , n. 15).

19. Id.

20. Le roman de Mme de Duras remporta un vif succès, alors qu'en 1822, la pièce intitulée Le nègre, proposée au théâtre de la Gaité par le jeune Balzac ne fut jamais représentée. Voir Gilles Castagnes, «Érotisme, violence, subversion et monstruosité dans Le Nègre de Balzac: une pièce inacceptable? », Littératures, 69, 2013, [en ligne], et «No Longer Simply Black and White: Adaptation and the Representation of Female Vulnerability in Balzac's Le Nègre », Lingua Romana, 11, 1, 2013, p. 78-102.

21. Felix Mengin a publié en 1823 Histoire de l'Égypte sous le gouvernement de Mohammed-Aly sous les auspices de «son Excellence M. le Vicomte de Chateaubriand, Pair de France, Ministre Secrétaire d'État au Département des Affaires Etrangères ». 
Vice-Roi, a reçu de ce prince l'accueil le plus flatteur. - je charge cet Officier, de remettre Lui-même à leur E.E. les Ministres des Finances, de la Marine, de la Guerre, et des Affaires Etrangères, les présents de S.A. le Vice-Roi $[\ldots]^{22}$.

On peut donc raisonnablement supposer que Morgan fut offert à Chateaubriand, au même titre qu'un cadeau diplomatique, lors de l'un de ces envois orchestrés par Drovetti. Chateaubriand lui-même dit son intention d'envoyer un cadeau à Méhémet-Ali dans une lettre du 24 mars $1823^{23}$. Avait-il déjà reçu Morgan à cette date? Le fait que Chateaubriand évoque Morgan dans un texte relatant sa vie publique en tant que ministre des Affaires étrangères laisse à penser qu'il voulait situer la présence de l'enfant dans ce contexte. Mentionnons cependant que Morgan arrive en France alors que l'on a discuté au Congrès de Vérone de l'abolition de la traite des noirs. «Toutes les puissances répondirent que la traite des nègres était abominable, qu'elles étaient prêtes à concourir aux mesures jugées exécutables, pour assurer l'abolition totale de ce commerce $[. . .]^{24} »$. Et, dès son entrée en fonction en tant que ministre des Affaires étrangères, Chateaubriand écrit le 31 janvier 1823 au comte de Beaurepaire, chargé des affaires de France à Constantinople :

J'ai l'honneur de vous adresser la copie d'une Ordonnance du Roi, qui défend, sous des peines sévères, à tout armateur et capitaine français d'employer ou d'affréter leurs navires pour transporter des esclaves.

Ces coupables désordres ont été particulièrement remarqués soit dans les mers du Levant, soit dans les parages d'Égypte et de Barbarie; et les guerres civiles qui désolent ces contrées ont souvent offert l'occasion d'y

22. Bernardino Drovetti, Epistolario (180o-1851), éd. Silvio Curto, en collaboration avec Laura Donatelli, Milan, Editoriale Cisalpino - La Goliardica, 1985, lettre 220 .

23. Chateaubriand écrit à Marcellus, premier secrétaire d'ambassade à Londres, le 24 mars 1823: "J'ai quelque fantaisie d'envoyer ce chef-d'œuvre, dont je suis peu digne, à Méhémet-Ali, afin que, après avoir porté l'ambassadeur de France sur les pavés de Londres, il roule le pacha d'Égypte sur les sables du Nil. Les voitures ont aussi leurs destinées! Habent sua fata... » (Politique de la restauration en 1822 et 1823 , Paris, Jacques Lecoffre et Cie, 1853, p. 175).

Il s'agit de la voiture de gala confectionnée pour l'audience personnelle de Chateaubriand, alors ambassadeur à Londres: cadeau prestigieux qu'il souhaite envoyer au pacha d'Égypte.

24. François-René de Chateaubriand, Le congrès de Vérone, Leipzig, Blockhaus et Avenarius, 1838 , p. 53. 
abuser du droit de la force. Les individus tombés au pouvoir de leurs ennemis sont traités comme esclaves et transportés sur d'autres rivages pour y être vendus. Le Roi a voulu empêcher qu'un acte si contraire aux lois de l'humanité ne fût protégé par ses sujets et couvert de son pavillon ${ }^{25}$.

Dans ces conditions, comment expliquer la présence de Morgan sur le sol français? S'il n'est pas esclave, quel est donc son statut social et juridique?

Dans Le congrès de Vérone, l'enfant nous est d'abord présenté dans la pure tradition de l'Ancien Régime: il est assimilé à l'animal exotique domestiqué («nous n'avions pas [...] un lion familier [...] mais [...] un négrillon ») à vocation ludique : il distrait ici de l'ennui et des désagréments des sollicitations importunes à l'hôtel des Affaires étrangères ${ }^{26}$. Dans un deuxième temps, son ascendance aristocratique permet une comparaison avec le duc de Bordeaux, son camarade de jeux occasionnel, puisqu'ils sont tous deux rois sans trône («Ce petit roi noir, à l'instar du petit roi blanc»). Le récit de Chateaubriand est publié 15 ans après les faits. Au moment de la narration, Morgan n'est plus de ce monde et le duc de Bordeaux, devenu comte de Chambord en 1830 et considéré comme le roi Henri $\mathrm{V}$ par les légitimistes, est en exil avec sa famille. Chateaubriand semble implicitement reconnaître le dommage causé à Morgan par le déracinement dont il a été l'objet. On apprend aussi dans ce texte publié en 1838 que Morgan meurt à la fleur de l'âge, comme Ourika, mais on ne sait ni de quoi, ni quand au juste ${ }^{27}$.

Quelles sont les traces laissées par Morgan après 1823?

25. François-René de Chateaubriand, Correspondance générale, éd. Louis Thomas, Paris, Librairie ancienne Édouard Champion, 1913, t. IV, p. 85.

26. Comme le montre la lettre de Drovetti au comte d'Augier, les présents envoyés sont pour la plupart des animaux exotiques. C'était le cas depuis bien longtemps. Ourika elle-même fut assimilée aux animaux ramenés par le chevalier de Boufflers. Lettre 200: «Ce 19 [juillet 1786]. Il me reste une perruche pour la reine, un cheval pour M. le maréchal de Castries, une petite captive pour M. de Beauvau, une poule sultane pour le duc de Laon, une autruche pour M. de Nivernois [...]» (Lettres d'Afrique à Madame de Sabran, éd. François Bessire, Paris, Actes Sud, 1998, p. 170). La petite captive n'est autre qu'Ourika.

En 1826, Charles X recevra un girafon du vice-roi d'Égypte.

27. Signalons que l'édition critique du Congrès de Vérone, parue en 2014, n’apporte aucune information sur la présence de Morgan en France. 
En août 1824, on le retrouve en Suisse avec Mme de Chateaubriand ${ }^{28}$. Voici ce que rapporte le fils de Louis Caumont, directeur de pensionnat, à qui Mme de Chateaubriand s'était adressée, espérant qu'il prenne en charge l'éducation de Morgan:

En 1824, Mme de Chateaubriand, passant quelques mois à Neuchâtel [...], fit un jour appeler mon père auprès d'elle et lui proposa de prendre en pension chez lui un joli petit nègre de sept ans, dont un chef africain avait fait présent à son mari. Ce petit nègre était rempli d'esprit, mais ignorant les premiers principes de l'instruction au-delà de la lecture. Cette bonne dame pensait qu'il ne serait pas déplacé au milieu de jeunes gens déjà instruits et beaucoup plus âgés que lui. Elle l'avait traité jusque-là en enfant gâté, le faisant habiller par un domestique, qui ne lui avait pas même appris à attacher ses souliers, et le couchant dans un lit de grand seigneur. Et elle pensait que cet enfant ne donnerait pas de peine, qu'il n'y avait pas à se gêner avec lui pour la nourriture, et qu'on pouvait tout uniment lui donner pour lit un sac de paille! Elle voulait qu'il fût élevé dans la religion catholique, et prétendait qu'il serait facile à mon père, qu'elle savait être protestant, de l'élever dans les croyances romaines. Bref, cette Mme la vicomtesse de Chateaubriand, femme du célèbre auteur du Génie du Christianisme, alors exilé de France par les Bourbons, elle-même se jugeant une femme supérieure, déraisonnait. Le petit négrillon resta chez elle.

Nos pensionnaires le voyaient souvent; il venait jouer avec eux presque tous les soirs, à la promenade du Crêt, et c'était pour eux un divertissement de s'entretenir avec lui; les plus jeunes n'auraient pas demandé mieux que de le voir entrer dans le pensionnat; mais dans quelle catégorie aurait-il fallu le placer ${ }^{29}$ ?

Mme de Chateaubriand tente de faire entrer Morgan dans ce pensionnat de Neufchâtel en vain. On comprend les raisons du refus de Caumont qui, par deux fois, mentionne la difficulté qu'éprouverait le petit négrillon à trouver «place» au sein du pensionnat. Ces propos font écho à la plainte d'Ourika qui dit dans le roman: «Cette société où j'étais déplacée ${ }^{30} »$. On apprend également que Morgan attisait l'intérêt des pensionnaires pour lesquels il était un divertissement, une

28. Mme de Chateaubriand séjourne à Neuchâtel du $1^{\text {er }}$ août au 22 octobre 1824 . Voir Mémoires d'outre-tombe, éd. Maurice Levaillant et Georges Moulinier, Paris, Gallimard, coll. «Bibliothèque de la Pléiade», 1951, t. II, p. 1068.

29. Frédéric Caumont, «Autobiographie et souvenirs», Musée neuchâtelois. Recueil d'histoire nationale et d'archéologie, 30, 1893, p. 114-115, je souligne.

30. Mme de Duras, Ourika, op. cit., p. 10. 
curiosité exotique. Cette impression est relayée par une note de l'éditeur Philippe Godet: «Ce négrillon, qui répondait au nom de Morgen $[$ sic $],[. .$.$] a laissé un vif souvenir à ceux qui l'avaient connu. Un de nos$ abonnés, qui était enfant en 1824, nous a raconté qu'il s'est souvent baigné au Crêt avec le groom de Mme de Chateaubriand: les gamins s'amusaient à le frotter et le savonner "pour voir s'il était bon teint"

Le 22 octobre 1824, la vicomtesse de Chateaubriand et Morgan rentrent à Paris. Un an plus tard, le 31 décembre 1825 , Chateaubriand écrit à la comtesse de Castellane :

Voici comment va se passer mon premier jour de l'an. Comme je suis tout seul dans le monde, je fais venir ma famille, c'est-à-dire Morgan, auquel Mme de Chateaubriand a acheté un jacko. Le pauvre garçon qui n'a que moi ici-bas mangera beaucoup, dormira longtemps, presque toute la journée; il est tout nu ou à peu près, car je ne suis pas bien riche et ses habits ne lui durent pas quinze jours. On lui mettra une de mes vieilles redingotes, un pantalon trop grand pour lui, un bonnet de Mme de Chateaubriand et des pantoufles à Joséphine, et il sera comme s'il était encore en Abyssinie chez le roi son père. Quelle destinée encore pour ce malheureux petit nègre: être jeté dans mes bras du bout du monde, moi, qui ne peux rien pour lui. Il y a dans ce dessein de la Providence quelque chose qui me touche: le pauvre orphelin est mal tombé 32 .

Dans ce passage pour le moins surprenant, Chateaubriand nous présente d'abord Morgan comme s'il était son fils adoptif. Peut-on y voir, alors qu'il a 57 ans, une certaine nostalgie de l'enfant qu'il n'a jamais eu ni vraiment voulu? Ne confie-t-il pas dans ses Notes et pensées: «Haine des enfants et pourtant profond désir d'en avoir d'une femme aimée $^{33} »$. Une femme aimée comme la comtesse de Castellane à qui il écrit le 5 octobre 1823 , alors que Morgan venait d'entrer dans sa vie: «[...] je perds cette nuit que j’aurais passée dans tes bras! [...] Tu

31. Frédéric Caumont, «Autobiographie et souvenirs», art. cit., p. 115.

32. François-René de Chateaubriand, Lettres à la comtesse de Castellane, publiées par la comtesse Jean de Castellane, Paris, Plon, 1927, p. 82-83; Correspondance générale, éd. Agnès Kettler et Pierre Riberette, Paris, Gallimard, 2004, t. VII, p. 116117 , je souligne.

33. François-René de Chateaubriand, Notes et pensées, éd. Mme la comtesse de Durfort, Bulletin de la Société Chateaubriand, 4, 1934, p. 39. 
m’aurais donné un fils; tu aurais été la mère de mon unique enfant ${ }^{34}$ ». Mais l'inclusion de Morgan dans le cercle familial est vite démentie dans la suite de la lettre où Chateaubriand s'avoue incapable de prendre complètement en charge «le pauvre orphelin». Il le dépeint dans un accoutrement grotesque, le renvoyant figurativement d'où il vient... l'Abyssinie. On verra plus loin comment il compte s'y prendre pour le renvoyer sur la terre de ses ancêtres. En attendant, Morgan vit dans l'entourage de Chateaubriand et celui-ci demande le 28 septembre 1826, à l'auteur d'Ourika:

Voulez-vous servir de marraine à Morgan? Je serai le parrain. Si vous ne pouvez l'être en personne, voulez-vous donner votre procuration à $\mathrm{M}^{\mathrm{de}}$ de Rauzan? Si vous acceptez l'une ou l'autre proposition voulezvous fixer l'époque du Baptême? Ce sera quand vous voudrez. [...] Il faut seulement que je sache quand il y aura baptême pour en prévenir l'archevêque. La cérémonie aura lieu dans la chapelle particulière. Vous sentez qu'il ne s'agit d'aucun frais et que le pauvre Morgan n'a besoin que d'être chrétien. Je ne puis lui donner de trésor que pour l'autre monde $e^{35}$.

Mme de Duras, malade, a accepté de devenir marraine par procuration puisqu'il lui écrit le 4 octobre: «Nous nommerons donc Morgan François Claire. Il sera baptisé le 19 à l'infirmerie jour où l'on célébrera la $\mathrm{S}^{t}$ Thérèse $^{36}$. Larchevêque de Paris fera le Baptême ${ }^{37}$ ». Baptiser Morgan, en faire un chrétien, est primordial attendu qu'il ne peut être heureux que dans l'autre monde. Le baptême n'est pas passé inaperçu puisqu'il fut annoncé dans le Journal des débats le 21 octobre:

34. François-René de Chateaubriand, Correspondance générale de Chateaubriand, éd. Louis Thomas, Paris, Librairie ancienne Édouard Champion, 1924, t. V, p. 25, cité dans le Bulletin de la Société Chateaubriand, 4, 1934, p. 39. "Pour la première fois il envisage de se perpétuer et souhaite qu'elle [Cordelia de Castellane] lui donne un fils qui soit le gage de leur amour et conserve leur mémoire» (Jean-Claude Berchet, Chateaubriand, Paris, Gallimard, 2012, p. 700).

35. François de Chateaubriand, «Lettres inédites sur le baptême du petit noir Morgan", dans Pierre Bergé et Associés, La duchesse de Duras et ses amis, Chateaubriand, op. cit., p. 121, je souligne.

36. Mme de Chateaubriand fit œuvre de charité. Elle fonda l'infirmerie MarieThérèse en 1819 et y accueillit les femmes de condition ruinées et les prêtres âgés.

37. François de Chateaubriand, «Lettres inédites sur le baptême du petit noir Morgan ", dans Pierre Bergé et Associés, La duchesse de Duras et ses amis, Chateaubriand, op. cit., p. 121. 
On célèbrera à l'infirmerie de Marie-Thérèse, rue d'Enfer, $n^{\circ} 86$, dimanche prochain, à trois heures, la fête de la patronne de l'établissement. M. l'archevêque de Paris administrera le baptême à un jeune Nègre âgé de neuf ans. Cet enfant, né en Abyssinie, a échappé presque seul, il y a quatre ans, au massacre que le pacha d'Égypte fit de la tribu dont son père étoit chef ${ }^{38}$.

Le lendemain, Chateaubriand écrit à Mme de Cottens ${ }^{39}$ :

Hier nous avons eu la cérémonie de notre Sainte-Thérèse, à l'Infirmerie, et nous avons baptisé un pauvre petit Noir, que la Providence m’a envoyé quand j'étais ministre. Il est mal tombé et son père blanc n'est guère plus riche que son père noir: mais enfin, il m’est donné de Dieu et du malheur, je lui serai bon parent ${ }^{40}$.

Chateaubriand ne mentionne pas cette fois le nom du «pauvre petit Noir». Pourtant Morgan s'est enrichi depuis la veille de ses prénoms de baptême; il s'appelle désormais Morgan François Claire. Chateaubriand semble apprécier l'opposition noir-blanc pour établir un parallèle entre individus vivant une situation difficile. On se souvient du parallèle établi dans le Congrès de Vérone entre le petit roi noir (Morgan) et le petit roi blanc (le duc de Bordeaux), tous deux privés de leur trône. Ici Chateaubriand évoque plus, dans le comparatif père blanc/père noir, ses propres difficultés financières qu'un statut paternel.

Le journal la Quotidienne rendit compte de la cérémonie le 24 octobre:

M. l'archevêque a prononcé dans cette occasion un discours rempli de cet esprit de charité qui anime ce prélat; il l'a terminé par une allocution dont voici à peu près les termes: Et vous, âmes généreuses (faisant allusion à $\mathrm{M}$. et à $\mathrm{M}^{\mathrm{me}}$ de Chateaubriand) qui avez adopté cet enfant $d u$

38. Journal des débats politiques et littéraires, samedi 21 octobre 1826, p. 3, cité par Gustave Lanson, Revue universitaire, 12, 1, 1903, p. 173.

39. Chateaubriand fait la connaissance de Mme de Cottens pendant un séjour à Lausanne en 1825 .

40. [Arthur de Cazenove] Saint-Quirin, «Une correspondance inédite de Chateaubriand (1826-1836). Lettres à $\mathrm{M}^{\mathrm{me}}$ de Cottens, née Laure de Cazenove d'Arlens ", Le correspondant, 25 août 1901; François-René de Chateaubriand, Correspondance générale, t. VII, op. cit., p. 219-220, je souligne. Cette correspondance générale comprend jusqu’à présent 9 volumes. À noter que le t. VI de la correspondance générale qui couvre l'année 1823 et donc un grand nombre de courriers diplomatiques n'est pas encore publié. J'aurais peut-être pu y trouver une référence à l'arrivée de Morgan en France. 
malheur sans être vous-mêmes dans la prospérité, le temps des tribulations finira; vous n'abandonnerez point l'enfant que la Providence vous a envoyé, et le Père Commun de la grande famille des hommes vous récompensera de votre charitét ${ }^{4}$.

Le lendemain, le Journal de Paris imputera à la Quotidienne d'avoir fabriqué les propos de l'archevêque; propos qui seront confirmés par Chateaubriand dans sa lettre du 26 octobre au rédacteur de la Quotidienne. On y lit:

Je dois à la vérité de déclarer, Monsieur, que vous avez rapporté avec exactitude le passage du discours de M. l'archevêque de Paris. Le baptême d'un orphelin, à qui la religion rendait une famille, devait naturellement conduire le charitable prélat à parler des tribulations de la vie, et de la miséricorde d'un Dieu qui fait souvent sortir notre bonheur de ces épreuves ${ }^{42}$.

L'« $\operatorname{adoption}^{43}$ » de Morgan, suggérée dans les lettres à Mme de Castellane et Mme de Cottens, est mentionnée dans le discours de l'archevêque, mais on ne peut pourtant la prendre au pied de la lettre ${ }^{44}$ puisque « [Chateaubriand] avait l'intention de faire apprendre au petit Morgan l'état de charpentier et menuisier, et de l'envoyer au Brésil, où les ouvriers de ce métier étaient recherchés, et où un noir pouvait devenir citoyen. "Cela vaut mieux sans doute que d'être un mauvais sujet de laquais sur le pavé de Paris!"45 »

Si Morgan s'inscrit dans la famille chrétienne par son baptême, il ne peut cependant trouver «place» dans la société française. Et un avenir au Brésil ne se dessine pas non plus. En effet, en 1828, Chateaubriand obtient le poste d'ambassadeur à Rome. Morgan est du voyage puisque, d'après le récit du congrès de Vérone, il a été placé à la Propaganda Fide (Congrégation pour la Propagation de la Foi) qui «ne forme que des jeunes gens venus des pays infidèles ou hérétiques, et destinés à y

41. Journal des débats politiques et littéraires, samedi 21 octobre 1826, op. cit., p. $173^{-174}$.

42. Ibid., p. 174, je souligne.

43. Le terme «adoption» n'est pas à prendre ici dans son sens légal puisque selon la jurisprudence de l'époque, Morgan n'entre pas dans les conditions de l'adoption: l'adopté doit être français et majeur. Voir Agnès Fine, « Regard anthropologique et historique sur l'adoption. Des sociétés lointaines aux formes contemporaines", Informations sociales, 146, 2008, p. 8-16.

44. Je renvoie à la lettre à Mme de Castellane du 31 décembre 1825.

45. Gustave Lanson, Revue universitaire, 12, 2, 1903, p. 173. 
retourner pour porter la vraie foi ${ }^{46} \gg$. Et Chateaubriand est un fervent partisan de l'œuvre missionnaire ${ }^{47}$.

Le 16 octobre 1829, Chateaubriand, de retour à Paris ${ }^{48}$, écrit, probablement à Monseigneur Castracane, alors secrétaire de la Propagande :

Monseigneur,

C'est un devoir sacré pour Mde de Chateaubriand et pour moi, en quittant Rome, de vous remercier de vos bontés pour le pauvre petit orphelin que la Charité chrétienne vous a fait prendre sous votre protection. Nous espérons qu'il se rendra digne de vos bienfaits, qu'il apprendra de Votre Eminence les vertus nécessaires à la carrière apostolique et périlleuse qu'il doit parcourir un jour $[\ldots]^{49}$.

Morgan, le petit orphelin, a alors une douzaine d'années et son avenir est déjà tout tracé: il s'agit pour lui de passer de nombreuses années à Rome au séminaire de la Propaganda Fide afin de devenir un prosélyte de la «vraie foi» sur sa terre natale.

Morgan a donc passé 5 à 6 ans de sa vie auprès des Chateaubriand; le petit négrillon qui courait sous les fauteuils de l'hôtel des Affaires étrangères et amusait la galerie s'est vu à un âge précoce dicter un avenir auquel il a pourtant échappé puisqu'«il a rendu son dernier soupir à la primeur du jour, à une heure matinale comme sa vie», apprend-on dans le Congrès de Vérone. Dans ses Notes et pensées, écrites après $1830^{50}$, Chateaubriand confie: «Il a péri à cinq heures du

46. Félix Gennevoise, La Propagande. Notice historique, Rome, Impression polyglotte de la S.C. de la Propagande, 1875, p. 65. Pour des informations complémentaires sur le collège de la propagande, voir Jean-Joseph-François Poujoulat, Toscane et Rome. Correspondance d'Italie, Bruxelles et Leipzig, Meline, Cans et compagnie, 1840, Lettre XXI, avril 1829, p. 262-273.

47. Chateaubriand dit à propos des bienfaits des missions catholiques: «Dans moins de trois siècles, la seule Compagnie de Jésus a fourni plus de douze mille missionnaires, dont plus de 700 ont versé leur sang pour la foi; ajoutons tous ceux qui sont sortis depuis cette époque des autres ordres religieux, des Franciscains, des Dominicains, de la Propagande de Rome, des Lazaristes, des Pères de la société de Marie, de la Congrégation de Picpus, du séminaire français des Missions étrangères, et que l'on juge ensuite du nombre d'âmes que leurs travaux ont gagnées à Dieu; or, le prix d'une seule âme est si grand aux yeux de la foi, qu'il surpasse celui de tout cet univers: qu'il est donc glorieux d'être appelé à contribuer à une telle œuvre!» (Jean-Paul Migne, Encyclopédie théologique, Paris, J.-P. Migne, 1862, t. XXVII, col. 1094-1095).

48. Il a donné sa démission le 28 août 1829 .

49. Catalogue de ventes Drouot-Richelieu, jeudi 9 avril 2015, lettre autographe $\mathrm{n}^{\circ} 31$, p. 9 .

50. D’après Mme de Durfort qui les a publiées. 
matin, à une heure matinale comme sa vie: il semble qu'on ait voulu lui éviter la chaleur du jour ${ }^{51}$ ». Chateaubriand suggère encore une fois les difficultés qu'aurait rencontrées le «pauvre Morgan » s'il avait vécu. Chateaubriand n'évoque pas les circonstances de sa mort. En fait, Morgan était entré à la Propaganda Fide le 13 avril $1829^{52}$ et y est mort le 28 mars 1831 de phtisie, nous révèle l'unique document concernant Morgan Franciscus aux archives historiques de la Propaganda Fide ${ }^{53}$.

On ne peut refuser à Chateaubriand d'avoir été pétri de bonnes intentions et pourtant ses propos sont pour le moins déroutants. Que ce soit dans le Congrès de Vérone ou ses lettres à la comtesse de Castellane, Mme de Cottens ou Mme de Duras, il est difficile de

\section{Bulletin de la Société Chateaubriand, 4, 1934, p. 51.}

52. Je n’ai pas trouvé trace de Morgan dans les mémoires de Mme de Chateaubriand. Par contre, elle évoque dans le Cahier vert, en octobre 1829, 6 mois après l'entrée de Morgan à la Propagande: "Ces pauvres enfants grecs, abordant sur les côtes de France pour y être élevés aux frais du Gouvernement qui aurait pu en faire de bons sujets et en même temps de bons catholiques. Les pauvres enfants, dis-je, rongés de scorbut et se mourant, ont été renvoyés dans leur patrie sans qu'on ait voulu leur permettre de descendre à terre pour être soignés [...] » (Les cahiers de Madame de Chateaubriand, éd. J. Ladreit de Lacharrière, Paris, Émile-Paul, 1909, p. 233). Note de l'éditeur: "Au début d'octobre 1829, on apprit que le comte Saint-Léger de Bemporta, neveu d'Hyde de Neuville, chargé de racheter des esclaves grecs en Égypte, avait obtenu de la générosité du roi de France que ces cinquante orphelins, dont les parents avaient été massacrés à Missolonghi, fussent élevés en France. À leur arrivée, à Toulon, ils reçurent l'ordre de se rembarquer aussitôt et de regagner leur pays. Les journaux ministériels prétendaient qu'on devait les rendre à leur famille mais l'opposition critiquait amèrement le manque de charité du ministère » (id.).

53. Je remercie chaleureusement Mgr Luis Manuel Cuna Ramos (Archivista Storico presso Congregazione per l'Evangelizzazione dei Popoli) de m'avoir envoyé le seul document concernant Morgan retrouvé dans «Archivio Storico di Propaganda Fide» (SC Collegio Urbano Miscellanea, vol. 9). Je n’ai pas trouvé trace de la disparition de Morgan dans la correspondance de Chateaubriand. Par contre, dans une lettre à Mme de Cottens datée du 24 mars, soit quatre jours avant le décès de Morgan, il écrit: "C'est aujourd'hui même, Madame, que je publie une petite brochure où je demande pour le duc de Bordeaux un tombeau en France, après avoir réclamé il y a huit mois, sa couronne» (Correspondance générale, éd. Agnès Kettler, Paris, Gallimard, 2015, t. IX, p. 35). On se souvient qu'il associera les deux enfants dans leur malheur dans son Congrès de Vérone («Ce petit roi noir, à l'instar du petit roi blanc»). Pourtant, contrairement au duc de Bordeaux, Morgan semble bien avoir disparu de ses préoccupations en 1831. Il écrit le 3 octobre, à Mme Galitzine : "J'ai été soir et matin occupé d'une défense de mon pauvre petit Henri, qu'on veut proscrire à nouveau avec ses parents » (ibid., p. 92), et le 31 octobre à Mme de Cottens : «Voilà la brochure. Je suis ici à peu près le seul défenseur du malheur et de l'innocence d'un enfant. Ma réclamation ne produira rien et sera oubliée dans deux à trois jours. N'importe, j’aurai rempli un devoir» (ibid., p. 97). 
vraiment démêler ses sentiments. Il semble éprouver de la compassion pour le pauvre orphelin mais reconnait qu'il est mal placé pour assurer l'avenir et le bonheur de l'enfant. Il ne peut que s'engager à le faire entrer par le baptême dans la famille chrétienne. Chateaubriand ne croit pas au bonheur sur terre pour Morgan («le trésor est pour l'autre monde») ou pour «le pauvre nègre» en général. Et seule la religion peut ouvrir cet autre monde, ce qui explique son ardent soutien de l'œuvre missionnaire. Voici ce qu'il dit à la Chambre des pairs, à la séance du lundi 13 mars $1826^{54}$ :

À Dieu ne plaise que je veuille diminuer l'horreur qu'inspire la traite des noirs ${ }^{55}$; mais enfin je parle devant des chrétiens, je parle devant de vénérables prélats d'une Église naguère persécutée. Quand on arrache un nègre à ses forêts, on le transporte dans un pays civilisé; il y trouve des fers, il est vrai; mais la religion qui ne peut rien pour sa liberté dans ce monde, quoiqu'elle ait prononcé l'abolition de l'esclavage; la religion, qui ne peut le défendre contre les passions des hommes, console du moins le pauvre nègre, et lui assure dans une autre vie cette délivrance que l'on trouve près du réparateur de toutes les injustices, près du père de toutes les miséricordes ${ }^{56}$.

Morgan, le petit négrillon de Chateaubriand, dont on apprend l'existence presque par inadvertance, n'a pas ou peu retenu l'attention des biographes $^{57}$. Ghislain de Diesbach a le mérite de mentionner Morgan

54. Dans cette allocution, Chateaubriand s'en prend particulièrement au crime de la traite des blancs. Il écrit à la comtesse de Castellane: «[...] aujourd'hui je vous écris le cœur tout ému encore d'un succès que j’ai obtenu hier à la Chambre des pairs. J'ai eu l'honneur de faire passer un amendement pour défendre le trafic des esclaves dans le Levant; c'est-à-dire que les vaisseaux français ne pourront pas porter de rivage en rivage les femmes, les enfants des malheureux Grecs, devenus esclaves entre les mains des Turcs. En attachant ce grand acte de justice, d'humanité, de religion à mon nom, je ne crois pas avoir ressenti dans ma vie une joie plus pure et plus légitime» (Correspondance générale, t. VII, op. cit., p. 151-152).

55. Notons que «[...] le fils de l'armateur négrier malouin René-Auguste de Chateaubriand n'évoqua qu'en de rares occasions la traite et l'esclavage qui avaient permis à sa famille de devenir propriétaire du château de Combourg (Nelly Schmidt, Abolitionnistes de l'esclavage et réformateurs des colonies. 1820-1851. Analyse et documents, Paris, Karthala, 2000, p. 99).

56. François-René de Chateaubriand, «Opinion de M. le Vicomte de Chateaubriand sur le projet de loi relatif à la répression des délits commis dans les échelles du Levant ", dans CEuvres complètes de M. le Vicomte de Chateaubriand, Paris, Didot Frères, 1840 , t. IV, p. 21, je souligne.

57. André Maurois (1939), Henri Guillemin (1965), Jean-Paul Clément (1998), Ghislain de Diesbach (1998 et 2004) et Jean-Claude Berchet (2012). Joan Evans (1939), 
dans sa biographie, mais rien dans le texte n'explique ou ne commente sa présence dans l'entourage des Chateaubriand. La première occurrence révèle sa présence en Suisse auprès de Mme de Chateaubriand. «Elle est arrivée à Neuchâtel avec sa femme de chambre Joséphine et un négrillon, Morgan, qui, avec les deux chattes, égayait le bureau de Chateaubriand aux Affaires étrangères. Ce singulier trio a beaucoup étonné les populations $[\ldots]^{58} »$ : le biographe souscrit ici à la représentation classique, sous l'Ancien Régime, de l'enfant noir considéré au même titre que l'animal de compagnie. La deuxième occurrence ${ }^{59}$ reprend, en partie, le passage de la lettre envoyée à Mme de Castellane le 31 décembre 1825 , citée plus haut. Mais ce passage ne sert qu'à mettre en évidence la tristesse et le dénuement de Chateaubriand face à ses revirements de fortune depuis qu'il est éloigné du pouvoir ${ }^{60}$. De Morgan, il n'est pas réellement question.

On attribue les propos suivants à Chateaubriand en 1822 :

Tout m'ennuie; ma vie entière n'est qu'un long ennui; dès l'enfance j'étais indifférent à tout; j’ai voyagé sans voir, espérant chasser l'ennui qui revenait toujours, poussé par je ne sais quelle lassitude d'existence. Je n'ai rien observé avec intérêt. Tout passait devant mes yeux sans me piquer du désir de connaître; ma vie n’est qu'indifférence; je serais désolé d'avoir fait le mal; ce ne m'est pas un grand plaisir d'avoir fait le bien. La vertu m'est chère, mais c'est plutôt par raisonnement que par sentiment. Je ne m'attache à rien; je sers le roi de tout mon cœur, mais sans joie et sans goût. Mon existence est une contrainte perpétuelle. La vertu est une belle chose; mais il faut des caractères exprès pour en jouir. [...] Il y a des âmes à demi mortes; la mienne est née ainsi ${ }^{61}$.

dans sa biographie, mentionne la présence de Morgan à Neufchâtel en ces termes: «There was nothing to be done but follow her [Céleste], with Pilorge, the valet, the maid and a little negro boy whom an inconvenient admirer had presented to him. By the end of August Chateaubriand had joined her. In a day or two the weather broke; Céleste declared herself ill and the little negro looked plum-coloured with the cold» (Joan Evans, Chateaubriand: a Biography, Londres, Macmillan, 1939, p. 288-289, je souligne).

58. Ghislain de Diesbach, Chateaubriand, Paris, Perrin, 2004, p. 393.

59. Ibid., p. 402-403.

6o. Renvoi de Chateaubriand du ministère des Affaires étrangères, le 6 juin 1824 .

61. Antoine Fontaney (Lord Feeling), «Conversations de Chateaubriand», Revue des Deux Mondes, $1^{\text {er }}$ décembre 1834, p. 576, cité dans Jean-Paul Clément, Chateaubriand. Biographie morale et intellectuelle, Paris, Flammarion, 1998, p. 579, n. 9. "Sous le pseudonyme de Lord Feeling, Antoine Fontaney (1803-1837) avait publié dans la Revue des Deux Mondes du $1^{\mathrm{er}}$ décembre 1834 des "Conversations de 
Dans un tel état d'esprit, comment Chateaubriand aurait-il pu sérieusement intégrer dans son foyer l'enfant qui allait bientôt surgir dans sa vie? Morgan paraît devant lui, non en enfant désiré mais comme cadeau diplomatique (tel un animal exotique), cadeau que le ministre des Affaires étrangères ne peut sans doute pas refuser. Morgan ne fut donc pas adopté, quoiqu'on en dise ${ }^{62}$. Si le jeune enfant fut comparé, dès son entrée à l'hôtel des Affaires étrangères, à un animal domestique, à l'instar des chats de Chateaubriand, on peut imaginer qu'il devint en grandissant plus encombrant que ces félins. Cet enfant, sans statut social ou juridique, sans place réelle dans l'entourage de Chateaubriand, ne pouvait espérer, si l'on en croit ce dernier, qu'une vie meilleure dans l'autre monde où il redeviendrait «la perle de sa mère ».

Chateaubriand" prétendument recueillies auprès de l'écrivain pendant son ambassade à Londres par un jeune Anglais de ses amis. Les propos rapportés peignent Chateaubriand tel qu'en lui-même [...]» (Correspondance générale, t. IX, op. cit., note 2 de la lettre 579 à François Buloz). État d'esprit de Chateaubriand confirmé par Marcellus: "Tout me lasse", me disait Chateaubriand un soir que nous revenions des solitudes du parc de Kensington, sa promenade favorite; "tout me lasse, et je vais partout en bâillant ma vie" " (Politique de la Restauration en 1822 et 1823, op. cit., p. 67, n. 1). On retrouvera la formule améliorée dans les Mémoires d'Outre-Tombe: «Tout me lasse; je remorque avec peine mon ennui avec mes jours, et je vais partout bâillant ma vie» (éd. Jean-Claude Berchet, Paris, Bordas/Classiques Garnier, 1989, t. I, p. 446).

62. Dans une note en référence à la lettre de Chateaubriand à la Propagande, les éditeurs de la Correspondance générale mentionnent «Morgan, le négrillon que Chateaubriand adopta quand il était aux Affaires étrangères" (Correspondance générale, éd. Agnès Kettler et Pierre Riberette, Paris, Gallimard, 2010, t. VIII, p. 614). 